\title{
Multi-Constrained QoS Opportunistic Routing by Optimal Power Tuning in Low Duty-Cycle WSNs
}

\author{
V. P. Jayachitra ${ }^{1 *}$, G. Geetha ${ }^{2 *}$, Durbaka Aishwarya ${ }^{1}$ \\ ${ }^{1}$ Department of Computer Technology, MIT Campus, Anna University, Chennai, India \\ ${ }^{2}$ Department of Computer Science \& Engineering, Jerusalem College of Engineering, Anna University, Chennai, \\ India \\ Email: "jayachitravp@annauniv.edu, "togeethamohan@gmail.com
}

Received 17 April 2016; accepted 5 May 2016; published 12 August 2016

Copyright (C) 2016 by authors and Scientific Research Publishing Inc.

This work is licensed under the Creative Commons Attribution International License (CC BY).

http://creativecommons.org/licenses/by/4.0/

(c) (i) Open Access

\section{Abstract}

Designing a multi-constrained QoS (Quality of service) communication protocol for mission-critical applications that seeks a path connecting source node and destination node that satisfies multiple QoS constrains such as energy cost, delay, and reliability imposes a great challenge in Wireless Sensor Networks (WSNs). In such challenging dynamic environment, traditional routing and layered infrastructure are inefficient and sometimes even infeasible. In recent research works, the opportunistic routing paradigm which delays the forwarding decision until reception of packets in forwarders by utilizing the broadcast nature of the wireless medium has been exploited to overcome the limitations of traditional routing. However, to guarantee the balance between the energy, delay and reliability requires the refinement of opportunistic routing through interaction between underlying layers known as cross-layer opportunistic routing. Indeed, these schemes fail to achieve optimal performance and hence require a new method to facilitate the adoption of the routing protocol to the dynamic challenging environments. In this paper, we propose a universal cross-layered opportunistic based communication protocol for WSNs for guaranteeing the user set constraints on multi-constrained QoS in low-duty-cycle WSN. Extensive simulation results show that the proposed work, Multi-Constrained QoS Opportunistic routing by optimal Power Tuning (MOR-PT) effectively achieves the feasible QoS trade-off constraints set by user by jointly considering the power control and selection diversity over established algorithms like DSF [1] and DTPC [2].

\section{Keywords}

Cross-Layer, Transmission Power Control, Latency Control, Energy Efficiency, Network Throughput,

${ }^{*}$ Corresponding authors.

How to cite this paper: Jayachitra, V.P., Geetha, G. and Aishwarya, D. (2016) Multi-Constrained QoS Opportunistic Routing by Optimal Power Tuning in Low Duty-Cycle WSNs. Circuits and Systems, 7, 2928-2939. 


\section{Multi-Hop Wireless Networks, Mission Critical Applications}

\section{Introduction}

The WSN imposes different QoS requirements on different applications under limited power resources. WSNs that are used for mission-critical applications such as health monitoring for critical patients, emergency evacuations, and disaster warning systems are expected to detect fast changing events to meet real-time quality constraints including network throughput, delay and power efficiency. Hence designing a routing protocol for mission critical applications expect a balance between QoS, in order to deliver a guaranteed level of service for such applications.

Traditional QoS-aware routing protocol focuses on either delay or energy or reliability and are not suitable for the dynamic environment. Recently, an opportunistic routing is widely used, which exploits the robustness despite the unreliable transmission links of the WSNs. Also, the broadcasting nature of the wireless medium in opportunistic routing requires no routing table maintenance in contrast to deterministic routing. In order to achieve better routing performance for challenging environments, the opportunistic routing is exploited to optimize QoS constraints across non-adjacent layers, thus introducing the cross layer opportunistic routing.

The PHY(Physical)-Aware opportunistic routing, MAC(Medium Access Control)-Aware opportunistic routing and PHY-MAC aware opportunistic routing protocols improve the QoS of WSN by considering the underlying Physical and MAC layer parameters [3]. These approaches still do not achieve best performances and fails to address many issues like selection of feasible forwarder candidate set and support for both slow and fast fading channel conditions (i.e. opportunistic routing performs well under fast fading conditions). Hence, an intelligent universal cross-layer opportunistic routing protocol is needed to address the given stringent multi-constraints in WSNs.

This work introduces an efficient cross-layer opportunistic routing that considers power control and selection diversity to select the optimal forwarder set to draw the balance between QoS constraints despite fast/slow fading channel environment. In the proposed work, the relay node can adaptively adjust the forwarding policy dynamically to meet different traffic loads by optimally tuning its transmission power. However, the delay in unpredicted traffic environments can only be tackled by MAC protocols [4]. The contention-free MAC protocols like TDMA (Time Division Multiple Access) were not sensible to dynamic topology and hence contentionbased (Synchronous and Asynchronous) MAC protocols are good for dynamic network environments. The asynchronous MAC protocol significantly increases the one-hop delay due to the decoupling nature of the communicating network node. The contention-based synchronous, duty-cycle scheduling coordinates by exchanging the active and sleep period among nodes in the network, which causes control overhead.

Based on the analysis and observation, this paper introduces a cross-layer opportunistic routing in low duty-cycle scheduling for multi-constrained QoS. The combination of these two mechanisms can ensure better performance as the relay node in opportunistic routing can optimally select the efficient active forwarder set by tuning the transmission power level of it to meet the user-specified QoS bound. The objective of the work is to design a communication protocol to guarantee a trade-off among multi-constrained QoS in WSNs. The contributions of this work are listed as follows.

1) To adapt the new challenging dynamic environment like disaster management system etc.

2) To support slow or fast fading condition by combining opportunistic and selection diversity routing scheme.

3) To propose a universal cross-layer design such that the designed network can guarantee the user specified QoS bound and successful data transmission through optimal transmission power control.

The rest of the paper is organized as follows. Section 2 presents the related work. In Section 3, Network environment and assumptions is specified and Design challenges of proposed work in discussed in Section 4 . The MOR-PT algorithm is presented in Section 5. The Experimental setup and resource utilization are presented in Section 6. Section 7 presents the simulation results, wherein the protocol is compared with the ETX, DSF, and DTPC protocol. The paper is concluded in Section 8 with focused Future work.

\section{Related Work}

There have been routing protocols designed keeping numerous applications and challenges in mind, but special 
emphasis has been paid to deliver a guaranteed level of QoS to applications. The QoS requirements can be specified in the form routing performance metrics, such as delay, throughput, jitter, etc. One of the main challenges in QoS trade-off provisioning is setting the bounds dynamically. Our objective is to bring a tradeoff between energy consumption, delay, and throughput based on bounds set by user which satisfy the application requirements. The efficient way to do this is by designing a cross-layer framework [5], where parameters of each layer such as RSSI, duty-cycle, transmission power are controlled dynamically. In this section, we discussed many power control and routing algorithms that aimed to achieve QoS trade-off in Wireless Sensor Networks.

\subsection{Power Control}

The goal is to minimize the energy consumption while ensuring the reliability of the network by controlling the power at an appropriate level rather than assuming at the uniform and fixed power level. In MQDPC (Distributed Power Control algorithm with Multi-QoS Constraints) [6], a heuristic Minimum spanning tree was constructed where a node can adjust its power to reach its parent was proposed to attain delay and throughput metric at the cost of energy. An energy efficient transmission power control in par with channel environment for IEEE 802.15.6 Body Area Networks is proposed in [7]. To support successful communication under fading environments by suppressing interference by controlling the transmission power control is proposed in Adaptive multi-channel transmission power control (AMC-TPC) [8]. To attain the user specified delay bound by tuning transmission power control using single-parent and multi-parent forwarding scheme is proposed in DTPC (Delay-Bounded Transmission Power Control) [2]. Note that all the above algorithms use traditional data forwarding scheme where data is forwarded to the pre-determined neighbor that substantially reduces end-to-end delay.

\subsection{QoS Routing}

To support dynamic environment contention free (Synchronous and Asynchronous) based MAC protocol plays the vital role in reducing energy in energy-constrained sensor nodes. Synchronous MAC protocol incurs more energy in exchanging schedule message between neighbors and enhances communication overhead. However, Asynchronous consume less energy as there is no slot exchange message between nodes but incurs the one-hop delay as the nodes were unaware about the wake-up time of their neighbor nodes. ETX (Expected Transmission Count Metric) [9] and PRR $\times$ D (Packet Reception Rate and Distance) [10] focus on throughput and end-to-end delay by employing traditional routing in low-duty-cycle wireless networks. The Dynamic switch-based forwarding(DSF) [1] uses traditional routing method in to optimize Data delivery ratio, delay and energy consumption by choosing the optimal forwarded from the set of first awakening forwarder node under low-duty cycle networks and results in retransmission if not succeed. However, recently opportunistic routing scheme under sleep-wake scheduling was introduced to choose energy-optimal path under statistically guaranteed delay [11]. In ASSORT (Asynchronous Sleep-Wake Scheduling and Opportunistic routing) [12], an opportunistic based routing under asynchronous scheduling to maximize the lifetime of the network was designed. However, a fundamental problem in multi-constrained QoS routing [13]-[15] where one seeks a path connecting a source node to a destination node that satisfies multiple QoS constraints, such as energy cost, delay and reliability is a challenging issue. Therefore, from the motivation of above-related work and to address various challenging issue, a novel routing protocol is proposed for WSNs, which can guarantee the data delivery with reduced energy consumption, communication latency and storage with less overhead.

\section{Network Model and Assumptions}

\subsection{Network Model}

We consider a static network composed of low-duty cycled nodes and an always on sink node. In order to consume reduced energy, the nodes are on asynchronous sleep-wake scheduling. A node is in either an active or a sleep mode. In active mode, it can sense, receive and send packets while in sleep mode, the data packets cannot be sent or received by them. We use Tunable-MAC protocol for handling MAC layer transmissions. To enhance network throughput and to avoid retransmission, opportunistic routing mechanism is used to select next hop in the given network, $\mathrm{N}(\mathrm{t})=(\mathrm{V}, \mathrm{L}(\mathrm{t}))$ where $\mathrm{V}=$ set of nodes in the given network and $\mathrm{L}(\mathrm{t})=$ set of wireless links at time $\mathrm{t}$. 


\subsection{Network Assumptions}

There are fixed number of discrete transmission power levels at which sensor node can transmit. Let each transmission power level be $\mathrm{P}_{i},\left(P_{1} \leq P_{i} \leq \mathrm{P}_{\mathrm{th}}\right)$, where $P_{1}$ is the minimum power level to ensure network connectivity and $P_{\text {th }}$ is often bounded by the maximum transmission power level of the node. All the sensor nodes are assumed to be in the power level $P_{d}$ which is the default power level taken as $-10 \mathrm{dbm}$. The link quality of a wireless channel is measured by the packet reception ratio (PRR). In low-duty-cycle sensor networks, data communication only happens when both the sender and receiver stay in the active mode. Since nodes in these networks are duty cycled, data traffic is assumed to be very low with less queuing delay.

Also, it is assumed that nodes at any time know

i) Packet reception ratio to the nodes in the forwarder set.

ii) Residual energy.

iii) Average delivery ratio for a given forwarding set "F" $\left(\mathrm{ADR}_{\mathrm{e}}(\mathrm{F})\right)$.

iv) Average transmission Delay for a given forwarding set "F" $\left(\mathrm{ATD}_{\mathrm{e}}(\mathrm{F})\right)$.

v) Average energy consumption for a given forwarding set " $F$ " $\left(\mathrm{AEC}_{e}(\mathrm{~F})\right)$ of the nodes that wake up next in their sensing area.

Table 1 shows the notations and explanations used in the proposed MOR-PT.

\section{Design Challenges}

Consider the given network N (V, L) consisting of n nodes. The sender node "e" should find the optimal forwarder set from the list of available forwarders $F=\left\{n_{i} \ldots, n_{n}\right\}$ with the specified user constraint on delay, energy and delivery ratio. The following factors were considered in selection of forwarder of the given node " $\mathrm{e".}$

i) To combine selection diversity and opportunistic routing to support in slow and fast fading channels. The selection diversity routing schemes select optimal relay candidates among multiple relay candidate that satisfy the user defined constraints on QoS. Among the optimal forwarders, opportunistic routing selects the next best awake relay nodes that are optimal in nature (i.e. delays the forwarding decision until data transmission is successful). Hence, the receivers at any hop are the relay node that satisfies user-specified multi-constraint QoS.

ii) Over time, the communication may result in temporary failure due to drop in Signal-To-Noise ratio or due to some other environmental factors. In that situation, the sender node cannot find optimal relay nodes from the current forwarder set. In that condition, the sender can take advantage of tuning to optimal transmission power to increase the forwarder set and optimizes forwarder set that satisfies multi-constraint QoS.

iii) To attain successful transmission of all data packets for mission critical applications by ensuring trade-off between delay and energy with low complexity overhead (i.e. $\log (n))$.

Table 1. Notations.

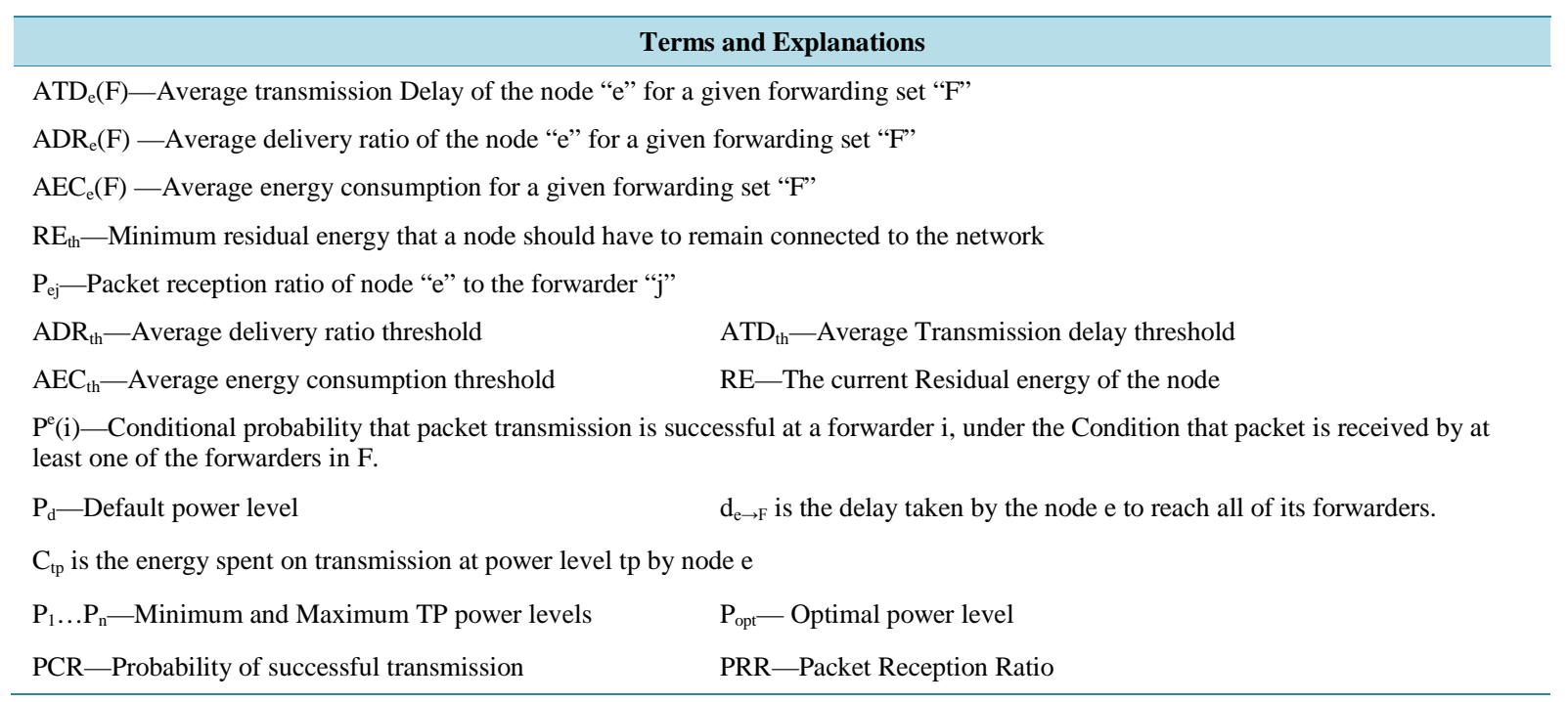




\section{Design of MOR-PT Strategy}

In this section, we present the design of MOR-PT to address the above design challenges. The DSF [1] and DTPC [2] work motivates our design MOR-PT and further improved in comparison to other routing strategies [2] [1] in following ways. First, we use the routing scheme to select the optimal forwarders satisfying multi-constraint (selection diversity) and to avoid retransmission by opportunistic forwarding and thereby improving the network throughput under controlled delay and energy consumption. Second, to enhance the robustness of communication under fast fading condition we enhance the MOR-PT metrics (AEC, ADR, and ATD) by adopting the sender to optimal transmission power dynamically with less complexity overhead rather than using the out-of-date predetermined metrics [1] [2] which suits only for the slow-fading condition. Figure 1 illustrates the framework of MOR-PT. Thus, the main process on MOR-PT includes the following phases,

i) Initialization Phase,

Initialization Phase

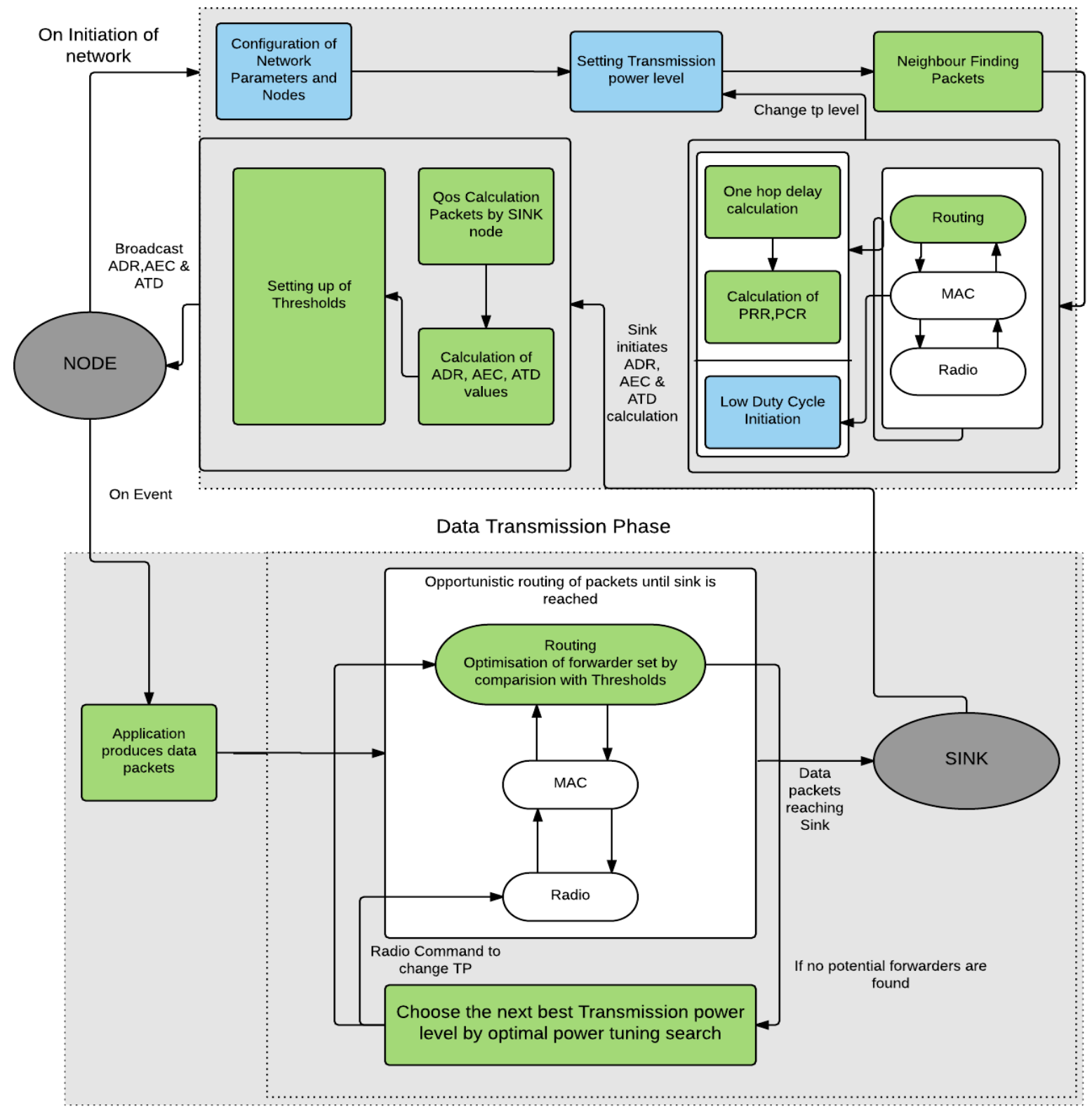

Figure 1. MOR-PT system overview. 
ii) MOR-PT routing phase, and

iii) Optimal Power selection phase.

The detailed explanation of each phase is explained in following sections.

\subsection{Initialization Phase}

Every node in the network does the initialization phase. Each node, at every power level, identifies the neighbors and in the same process calculates the packet reception ratio $\mathrm{P}_{\mathrm{ej}}$ and thereby $\mathrm{P}^{\mathrm{e}}(\mathrm{i})$ and stores it as a set in the MMAP map.

Assuming that there is only one single sink node (say s), for all the power levels, the forwarding set at sink node is empty, from [1], we can say that

$$
\operatorname{ADR}_{\mathrm{e}}(\Phi)=1, \quad \operatorname{ATD}_{\mathrm{e}}(\Phi)=0, \quad \operatorname{AEC}_{\mathrm{e}}(\Phi)=0
$$

The value of these is back propagated to the nodes which have this node as one of the forwarders. The nodes which get this information calculate the ADR, ATD and ATD values recursively at the power level $\mathrm{P}_{\mathrm{d}}$. The following section defines the method of calculation.

a) Average delivery ratio of the node "e" for a given forwarding set " $F$ " of size $n\left(A_{D} R_{e}(F)\right)$ is the product of the packet reception ratio between node e and forwarded $\mathrm{i}\left(\mathrm{P}_{\mathrm{ei}}\right)$ and Average delivery ratio $\left(\mathrm{ADR}_{\mathrm{i}}(\mathrm{F})\right)$ (at default TP )of the forwarder i to the sink node, calculated recursively.

$$
\operatorname{ADR}_{\mathrm{e}}(\mathrm{F})=\sum_{\mathrm{i}=1}^{\mathrm{n}} \mathrm{P}_{\mathrm{ei}} \mathrm{ADR}_{\mathrm{i}}
$$

b) Average transmission Delay of the node "e" for a given forwarding set "F" of size n $\left(\operatorname{ATD}_{e}(F)\right)$ is the product of $\mathrm{P}^{\mathrm{e}}(\mathrm{i})$ (Conditional probability that packet transmission is successful at a forwarder $\mathrm{i}$, under the Condition that packet is received by at least one of the forwarders in F) and Average transmission delay of the forwarder i to reach the sink, with their grand total (of all forwarders) added to the one hop delay experienced in reaching all of its forwarders at default TP.

$$
\begin{gathered}
\mathrm{P}^{\mathrm{e}}(\mathrm{i})=\frac{\mathrm{P}_{\mathrm{e}} \mathrm{ADR}_{\mathrm{i}}}{\operatorname{ADR}_{\mathrm{e}}(\mathrm{F})} \\
\operatorname{ATD}_{\mathrm{e}}(\mathrm{F})=\mathrm{d}_{\mathrm{e} \rightarrow \mathrm{F}}+\sum_{\mathrm{i}=1}^{\mathrm{n}} \mathrm{P}^{\mathrm{e}}(\mathrm{i}) \times\left(\operatorname{ATD}_{\mathrm{i}}\right)
\end{gathered}
$$

c) Average energy consumption for a given forwarding set "F" of size $n$ at default TP $\left(\mathrm{AEC}_{\mathrm{e}}(\mathrm{F})\right)$ is the product of packet reception ratio and Average energy consumption of the forwarder i to reach sink, with their grand total (of all forwarders) added to the energy lost in single transmission at that power level.

$$
\operatorname{AEC}_{\mathrm{e}}(\mathrm{F})=\mathrm{C}_{\mathrm{tp}}+\sum_{\mathrm{i}=1}^{\mathrm{n}} \mathrm{P}^{\mathrm{e}}(\mathrm{i}) \times\left(\mathrm{AEC}_{\mathrm{i}}\right)
$$

\begin{tabular}{|c|c|}
\hline $\mathrm{P}_{1}$ & $\begin{array}{c}\left\{\mathrm{n}_{1}, \mathrm{n}_{2}, \mathrm{n}_{3}, \mathrm{n}_{4}, \cdots, \mathrm{n}_{\mathrm{n}}\right\} \\
\left\{\mathrm{P}_{\mathrm{e} 1}, \mathrm{P}_{\mathrm{e} 2}, \mathrm{P}_{\mathrm{e} 3}, \mathrm{P}_{\mathrm{e} 4}, \cdots, \mathrm{P}_{\mathrm{en}}\right\} \\
\left\{\mathrm{P}^{\mathrm{e}}(1), \mathrm{P}^{\mathrm{e}}(2), \mathrm{P}^{\mathrm{e}}(3), \cdots, \mathrm{P}^{\mathrm{e}}(\mathrm{n})\right\}\end{array}$ \\
\hline $\mathrm{P}_{2}$ & $\begin{array}{c}\left\{\mathrm{n}_{1}, \mathrm{n}_{2}, \mathrm{n}_{3}, \mathrm{n}_{4}, \cdots, \mathrm{n}_{\mathrm{n}}\right\} \\
\left\{\mathrm{P}_{\mathrm{e}}, \mathrm{P}_{\mathrm{e} 2}, \mathrm{P}_{\mathrm{e} 3}, \mathrm{P}_{\mathrm{e} 4}, \cdots, \mathrm{P}_{\mathrm{en}}\right\} \\
\left\{\mathrm{P}^{\mathrm{e}}(1), \mathrm{P}^{\mathrm{e}}(2), \mathrm{P}^{\mathrm{e}}(3), \cdots, \mathrm{P}^{\mathrm{e}}(\mathrm{n})\right\}\end{array}$ \\
\hline$\cdots$ & $\cdots$ \\
\hline \multirow[t]{2}{*}{$P_{n}$} & $\begin{array}{c}\left\{\mathrm{n}_{1}, \mathrm{n}_{2}, \mathrm{n}_{3}, \mathrm{n}_{4}, \cdots, \mathrm{n}_{\mathrm{n}}\right\} \\
\left\{\mathrm{P}_{\mathrm{e}}, \mathrm{P}_{\mathrm{e} 2}, \mathrm{P}_{\mathrm{e} 3}, \mathrm{P}_{\mathrm{e} 4}, \cdots, \mathrm{P}_{\mathrm{en}}\right\} \\
\left\{\mathrm{P}^{\mathrm{e}}(1), \mathrm{P}^{\mathrm{e}}(2), \mathrm{P}^{\mathrm{e}}(3), \cdots, \mathrm{P}^{\mathrm{e}}(\mathrm{n})\right\}\end{array}$ \\
\hline & AEC, ATD, ADR at $P_{d}$ \\
\hline
\end{tabular}

Note that the delay and energy are added only once as in opportunistic routing, only one time the transmission is made. Therefore, the data structure for each and every node looks like Table 2. 


\subsection{MOR-PT Routing Phase}

In this section, we present MOR-PT protocol, a QoS Routing based on opportunistic routing, particularly for low duty-cycled sensor nodes. The process is depicted in Figure 1 as Data Transmission phase. MOR-PT optimizes the forwarding candidates among the nodes that hear packet transmission during opportunistic routing using the user's constraints (thresholds) on Delay, Energy, and Delivery ratio. The MOR-PT filters the nodes which are reachable under the current transmission power (identified through data structure) that satisfies the user's constraint. If the optimized forwarding set is empty, meaning there are no such nodes that satisfy the user's constraints, the transmission power is tuned to the optimal value found using optimal power selection search. A radio command is sent to the radio requesting the change of transmission power to the optimal value. This process is repeated until there is at least one forwarder in the optimized forwarder set. If the optimized forwarder set is large enough that it causes collision and energy loss, the forwarder set is restricted to optimal size, decided based on network size and density.

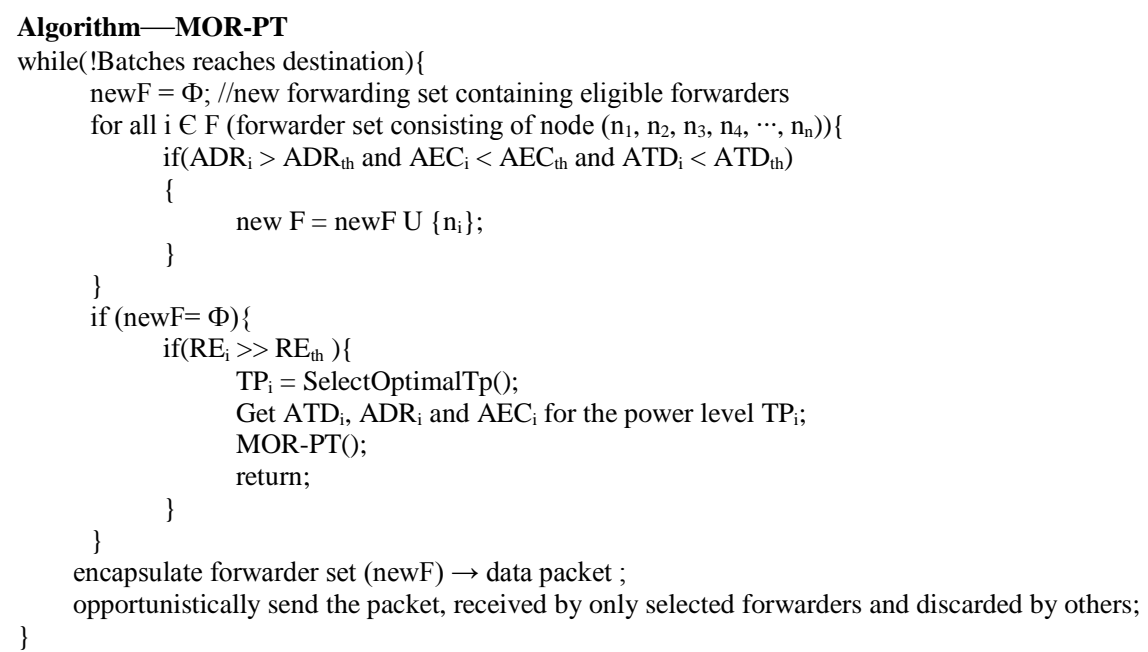

TRANSMISSION POWER LEVELS SEARCH SPACE

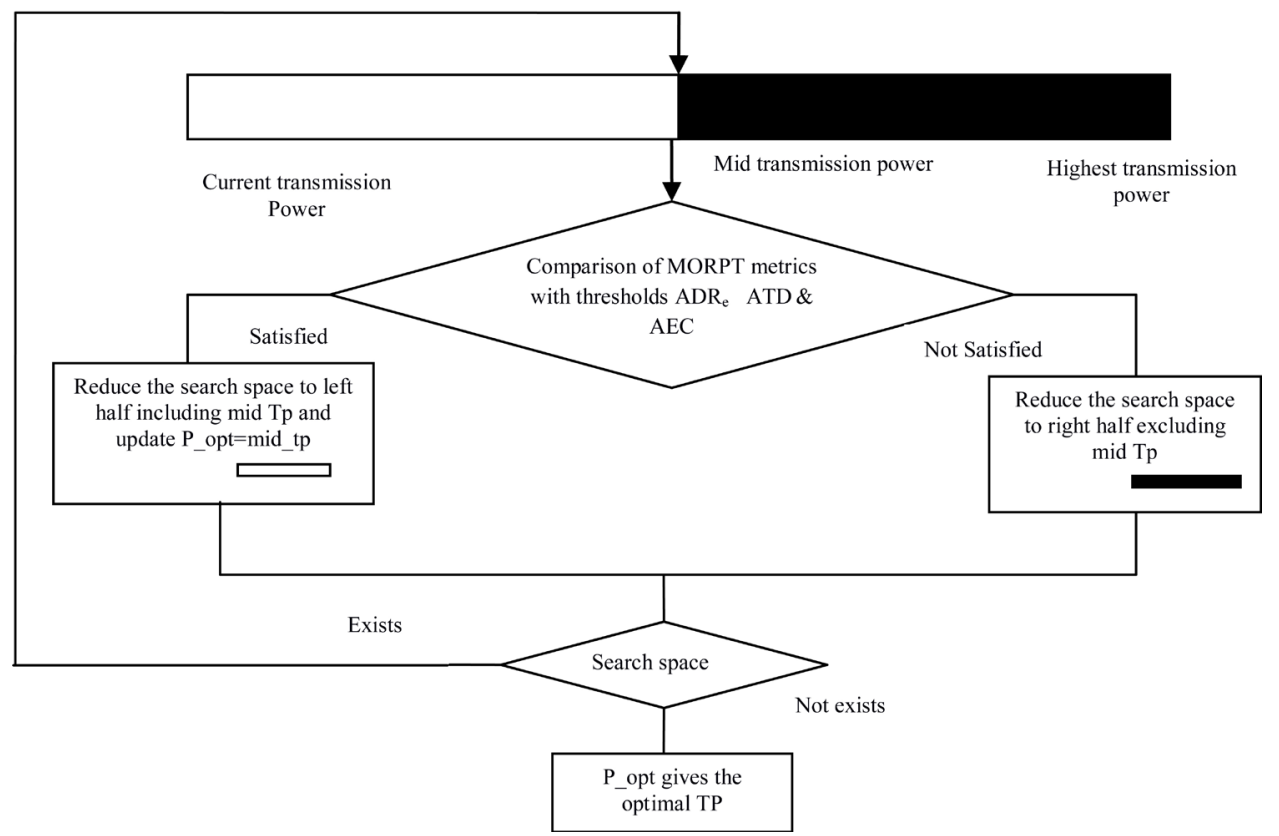

Figure 2. Flow chart of optimal transmission power selection scheme. 


\subsection{Optimal Power Selection Scheme}

The transmission power is tuned to the most optimal value evaluated using Select Optimal TP(). The Search space is the range of transmission power levels available for a node for transmission. The main theorem of Binary search states that search algorithm can be used if and only if for all $\mathrm{x}$ in $\mathrm{S}$ (Search space), $\mathrm{p}(\mathrm{x})$ implies $\mathrm{p}(\mathrm{y})$ for all $y>x$. If the Delay, Energy, and Delivery ratio constraints cannot be reached at a particular transmission power $\left(\mathrm{TP}_{\mathrm{i}}\right)$, then the same cannot be reached at a transmission power lower than $\mathrm{TP}_{\mathrm{i}}$ Hence, we can reduce our search space to the transmission power levels higher or equal to $\mathrm{TP}_{\mathrm{i}}$ as shown in Figure 2. Thus, the positive MOR-PT evaluation at transmission power $\mathrm{x}$ implies positive MOR-PT evaluation at transmission power $\mathrm{y}$ for all $y>x$. Thus, the application of binary search can be satisfactorily addressed in our problem statement. At each step of optimal power selection algorithm, the values of the ADR, AEC, ATD are calculated and used to check for the user's constraints and a decision is made to reduce the search space by one-half.

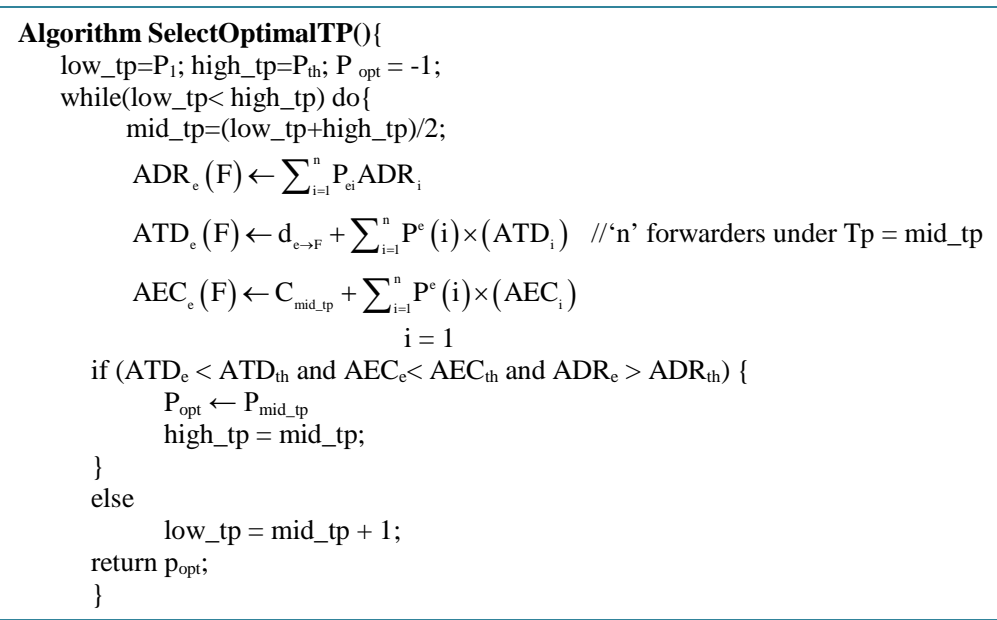

\section{Simulation}

We experiment our MOR-PT performance using Castalia [16], a realistic Wireless Sensor Network simulator that supports varied transmission power levels for an individual sensor node. We compared MOR-PT with the other related routing algorithms such as, 1) ETX and PRR*D improves data delivery ratio and end-to-end-delay using single potential forwarder in multihop wireless networks. 2) DSF chooses a single potential forwarder that wakes-up first from the set of forwarding nodes at each hop to improve energy, delay, and throughput. 3) DTPC that selects the potential forwarder by adjusting the power level to meet the desired Delay bound in a multihop wireless network. Throughput, Energy, and delay were the three performance metrics that we investigated in this evaluation by considering the experimental factors similar to the DSF and DTPC protocols to do a fair comparison.

\section{Simulation Setup}

We uniformly deploy 19 MICAz motes, with Sink in the center and 18 motes are arranged in the form of a matrix $3 \times 6$. The MAC protocol used is Tunable MAC that reflects realistic CC2420 radio model with tuned transmission power levels. Tunable-MAC is a Duty-Cycled that supports CSMA/CA scheme for transmitting data packets. The critical parameters such as Duty-Cycle, Beacon Interval, Listen Interval and MAC parameters can be dynamically tuned in Tunable-MAC. Based on the network size, i.e., $3 \mathrm{~m} \times 50 \mathrm{~m}$, the following (Table 3) are the different transmission power levels and the energy consumption spent by the CC2420 Radio when transmitting at the respective power level in Castalia.

Table 3. Radio transmission power levels.

\begin{tabular}{|c|c|c|c|c|c|c|c|c|}
\hline TX_dBm & 0 & -1 & -3 & -5 & -7 & -10 & -15 & -25 \\
\hline TX_mW & 57.42 & 55.18 & 50.69 & 46.20 & 42.24 & 36.30 & 32.67 & 29.04 \\
\hline
\end{tabular}


The default transmission power of all the nodes is set to least transmission power level of $-10 \mathrm{dbm}$. The $0^{\text {th }}$ node is declared as the sink node, which explains its placement in the center. The baseline power of the node is set to $6 \mathrm{dbm}$, general processing power used. The simulation is initiated by the sink node and thereby each node in the network builds the data table with the MOR-PT metrics. The data transmission is initiated by a random node that generates 1 to 100 packets. The packets are then forwarded opportunistically towards the sink node in accordance with MOR-PT metric. The node duty cycle of all nodes is set to 0.01 i.e. it listens for 1 unit time in 100. The following section discussed the proposed model QoS metrics with the counterpart under varied Duty-Cycle.

\section{MMAP}

The multi-constrained Map is established and maintained by each node that includes the details of the QoS constraint between surrounding neighbors. The get and set command are used for obtaining the muti-constrained parameters of the forwarder set. To attain memory tradeoff with algorithm metrics, we restrict the size of the map according to the node density of the network. The sendBeacon command is initiated by the sink node and broadcast its local MMAP for the other nodes in the network to compute their local MMAP at fixed power level. The receiveBeacon command is signaled to receive beacon message to establish their local MMAP. The MMAP for tuned power level was computed and broadcasted once if the critical finds no optimal forwarder that meets the required constraints.

\section{Result and Discussion}

In this experiment, the objective is to design a balanced lifetime-maximization through energy efficiency and Latency-Minimization with the guaranteed delivery ratio routing protocol. The proposed routing algorithm is studied under varied duty cycle but not under varied link quality and the stimulation results are discussed here. Since MOR-PT opportunistically selects the one that successfully received the packet from the possible set of potential forwarders selected by MORPT and hence need not to worry about the network dynamicity such as link quality or link breakages.

Figure 3 depicts that energy consumption of MOR-PT. The proposed algorithm consumes less energy compared to all other algorithms as there is no retransmission. Moreover, the optimal power tuning results in increasing the forwarder set and thereby selects the best forwarder that satisfies the minimum energy and other constraints (delay and throughput) assigned per hop path. The energy consumption of the MOR-PT increases as the duty cycle increases. This is because when duty cycle increases, more are the number awake forwarders that

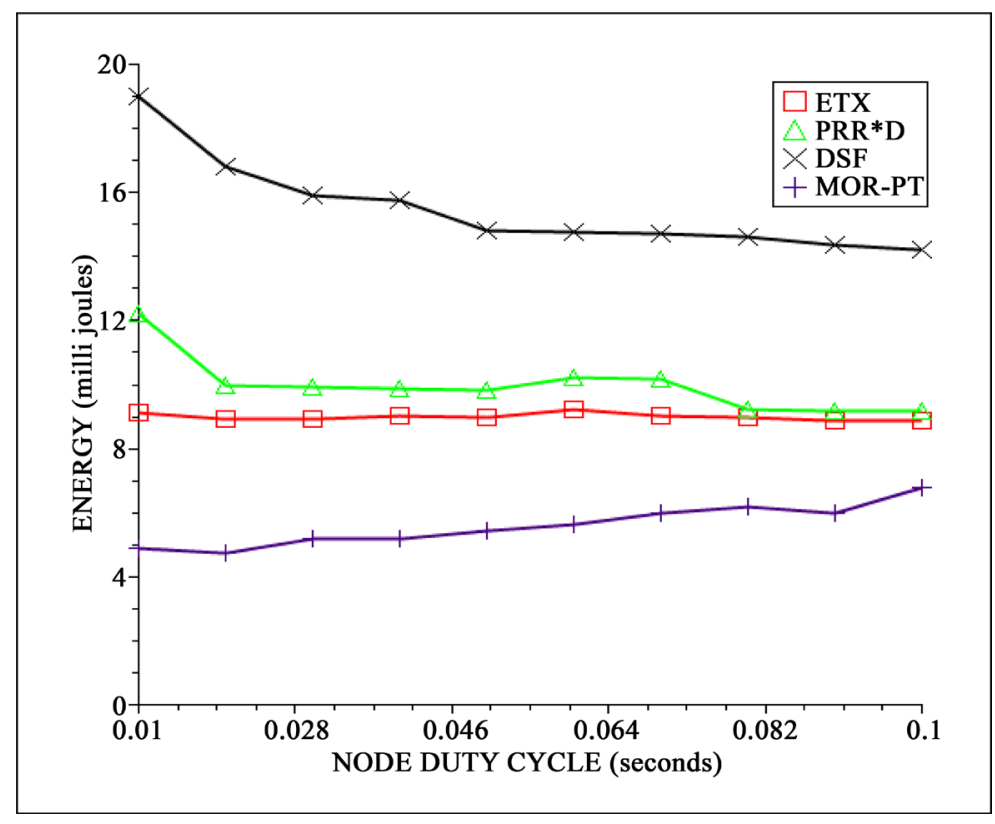

Figure 3. Duty cycle vs energy. 
satisfy the QoS constraints and hence overall energy consumption increases.

The ETX consumes higher energy than MOR-PT and remains constant throughout the varied duty cycle as the ETX elects the single next-hop node based on transmission count metric. The fluctuation in energy consumption of PRR*D was due to the varied distance factor in accordance with duty cycle. The DSF chooses the first awake node but not the best node and tries until retransmission count expires after which it tries for the next awaken forwarder for transmission. Hence DSF consumes higher energy than ETX.

From the Figure 4, the DSF under optimization of Delivery ratio, finds superior in attaining constant higher delivery ratio than other counterparts under different duty-cycle from 1 to 10 percent. This is because the DSF results in retransmitting the packets until the data delivery is successful irrespective of the increased sleep period. The proposed MOR-PT achieves higher delivery ratio in par with DSF for low data traffic as the duty cycle increases. Also opportunistic routing requires only one transmission for 1 hop as it ensures that there is at least one forwarder to receive the data packet. Hence it partly ensures delivery of all packets through some path determined by then best forwarder. MOR-PT makes use of opportunistic routing and chooses the potential forwarders by tuning the power level until it finds.

The delay of the network was also analyzed. Figure 5 shows the End-to-End Delay and a delivery ratio of MOR-PT, DSF, DTPC, and ETX. From the Figure 5 it has been seen that DTPC attains $95 \%$ at 15 s delay constraint. ETX attains increased delivery rate over time and delivers 55\% packet at $15 \mathrm{~s}$. DSF experienced lower delay than ETX and attains 98\% delivery ratio. The proposed protocol attains 100\% packet delivery ratio in accordance with used bound delay constraint. This is because the delay has no direct effect in accordance with the dynamic change of transmission power but can increase the neighbor set and then can optimally select the forwarder that satisfies user defined MOR-PT metrics.

From Figure 6, MOR-PT utilizes 38\% energy more than DTPC for initialization phase which is almost a onetime process. However the energy used for routing is $13 \%$ less than DTPC and $28 \%$ less than DSF, which is beneficial as the routing of data packets, is the recurring process. Since the transmission of the data is the main process which occurs repeatedly, the energy saved in routing nullifies and excels out the energy consumed in initialization and gives better performance than the later (DTPC).

\section{Conclusion}

MOR-PT, a new routing protocol that guarantees a tradeoff between energy costs, delivery delay and delivery

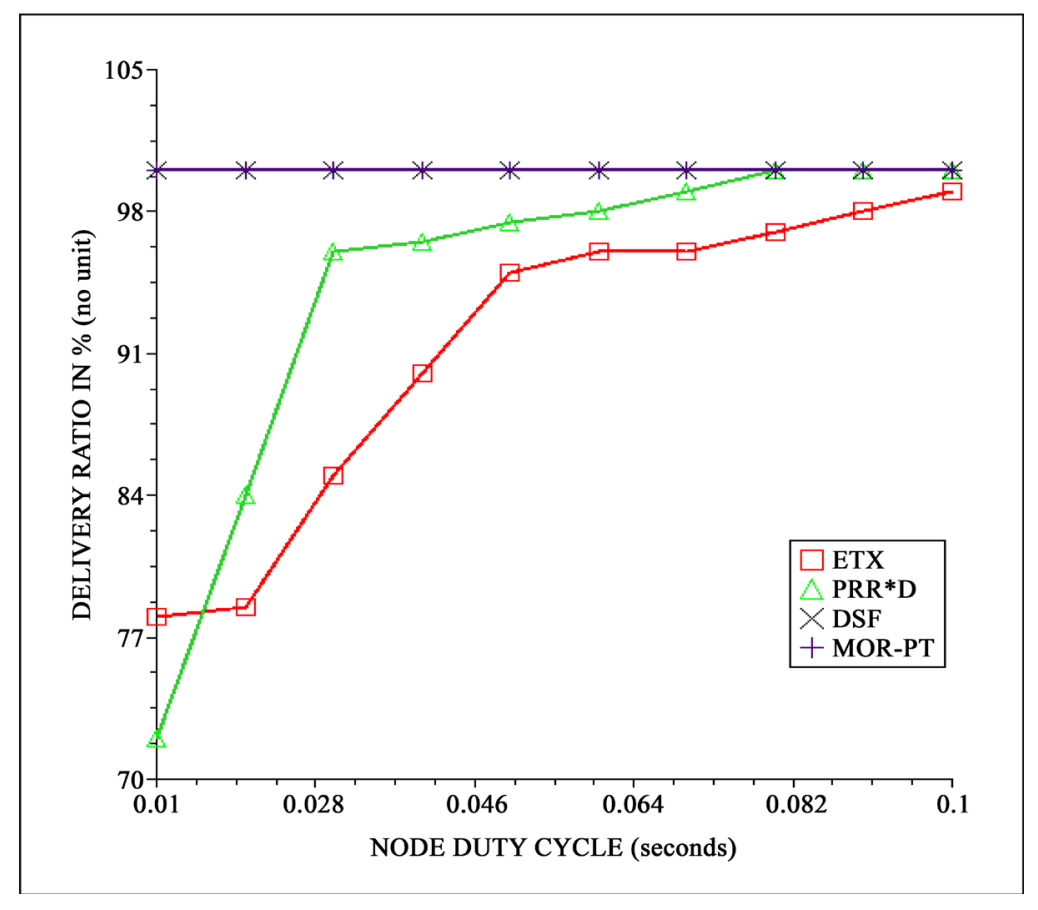

Figure 4. Duty cycle vs delivery ratio. 


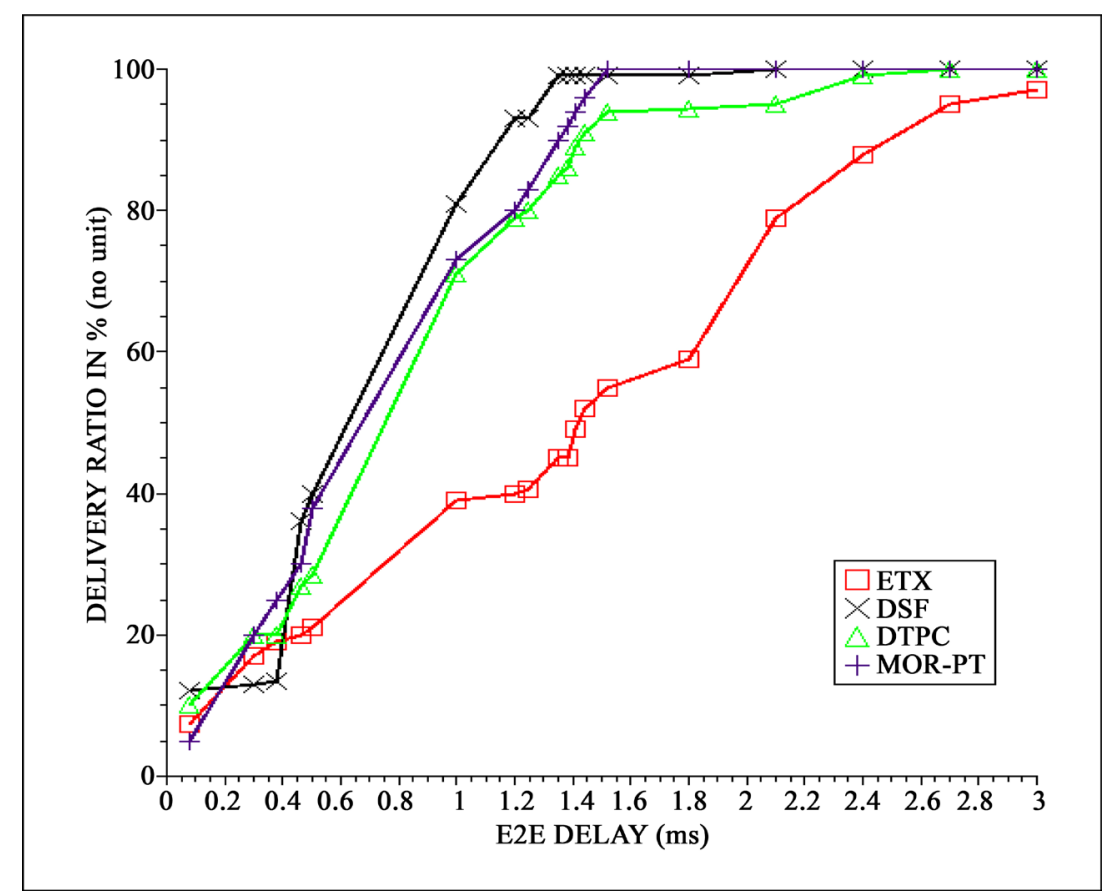

Figure 5. Delay vs delivery ratio.

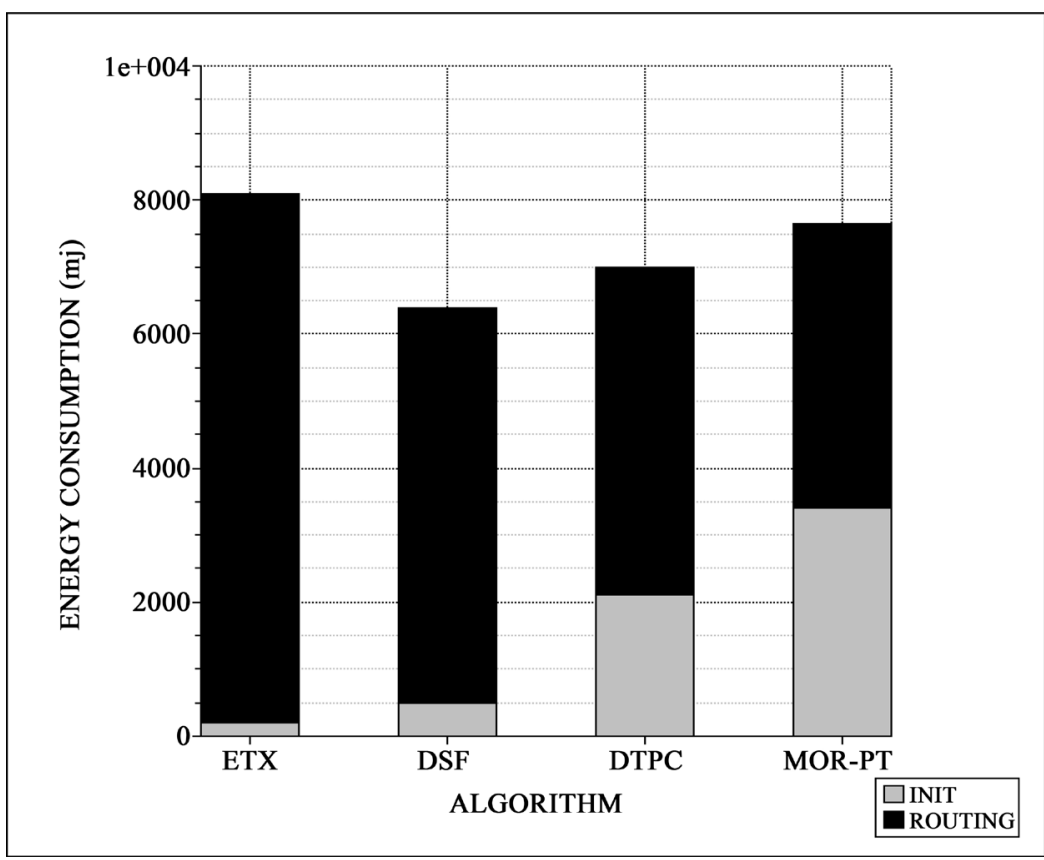

Figure 6. Initialization and routing phase energy consumption comparison.

ratio by satisfying the user set constraints and ensures universality. The MOR-PT metric efficiency is achieved by three steps, such as initialization, opportunistic routing, and optimal TP selection. The initialization step performs the formation of the MMAP with respect to MOR-PT metrics. The routing step optimizes forwarding set, based on the calculated local MMAP table of the sender node to transmit the data to the sink. The tradeoff between energy and delay and delivery ratio in a wireless sensor is achieved, by employing dynamic optimal power selection in routing phase. The simulation results show the superiority of the proposed scheme in maximizing the Multi-constraint QoS performance with less overhead by comparing it with the existing scheme, the DSF, 
and DTPC. In future, the MOR-PT algorithm can be combined with energy harvesting scheme to further enhance the energy efficiency and reduce delay by appropriately selecting the best and right forwarders under adaptive power control scheme.

\section{References}

[1] Gu, Y. and He, T. (2011) Dynamic Switching-Based Data Forwarding for Low-Duty-Cycle Wireless Sensor Networks. IEEE Transactions on Mobile Computing, 10, 1741-1754. http://dx.doi.org/10.1109/TMC.2010.266

[2] Fan, Z., Bai, S., Wang, S. and He, T. (2015) Delay-Bounded Transmission Power Control for Low-Duty-Cycle Sensor Networks. IEEE Transactions on Wireless Communications, 14, 3157-3170. http://dx.doi.org/10.1109/TWC.2015.2402681

[3] Chakchouk, N. (2015) A Survey on Opportunistic Routing in Wireless Communication Networks. IEEE Communications Surveys \& Tutorials, 17, 2214-2241.

[4] Huang, P., Xiao, L., Soltani, S., Mutka, M.W. and Xi, N. (2013) The Evolution of MAC Protocols in Wireless Sensor Networks: A Survey. IEEE Communications Surveys \& Tutorials, 15, 101-120.

[5] Al-Anbagi, I., Erol-Kantarci, M. and Mouftah, H.T. (2016) A Survey on Cross-Layer Quality-of-Service Approaches in WSNs for Delay and Reliability-Aware Applications. IEEE Communications Surveys \& Tutorials, 18, 525-552.

[6] Yang, H.-B. and Cai, W.-Y. (2008) Distributed Power Control Algorithm with Multi-QoS Constraints for Wireless Sensor Networks. IEEE International Conference on Networking, Sensing and Control, ICNSC, Sanya, 1031-1036. http://dx.doi.org/10.1109/icnsc.2008.4525368

[7] Gao, W.D., Jiao, B.L., Yang, G.L., Hu, W. and Liu, J.W. (2014) Transmission Power Control for IEEE 802.15.6 Body Area Networks. ETRI Journal, 36, 313-316. http://dx.doi.org/10.4218/etrij.14.0213.0220

[8] Ikram, W., Petersen, S., Orten, P. and Thornhill, N.F. (2014) Adaptive Multi-Channel Transmission Power Control for Industrial Wireless Instrumentation. IEEE Transactions on Industrial Informatics, 10, 978-990. http://dx.doi.org/10.1109/TII.2014.2310594

[9] De Couto, D.S.J., Aguayo, D., Bicket, J. and Morris, R. (2003) A High-Throughput Path Metric for Multi-Hop Wireless Routing. Proceedings of the 9th Annual International Conference on Mobile Computing and Networking (MobiCom'03), New York, 134-146. http://dx.doi.org/10.1145/938985.939000

[10] Seada, K., Zuniga, M., Helmy, A. and Krishnamachari, B. (2004) Energy-Efficient Forwarding Strategies for Geographic Routing in Lossy Wireless Sensor Networks. ACM SenSys’04, Baltimore, 3-5 November 2004. http://dx.doi.org/10.1145/1031495.1031509

[11] Cheng, L., Niu, J., Gu, Y., He, T. and Zhang, Q. (2015) Energy-Efficient Statistical Delay Guarantee for Duty-Cycled Wireless Sensor Networks. 12th Annual IEEE International Conference on Sensing, Communication, and Networking (SECON), Seattle, 46-54.

[12] Hsu, C.C., Kuo, M.S., Wang, S.C. and Chou, C.F. (2014) Joint Design of Asynchronous Sleep-Wake Scheduling and Opportunistic Routing in Wireless Sensor Networks. IEEE Transactions on Computers, 63, 1840-1846. http://dx.doi.org/10.1109/TC.2012.282

[13] Shah, G.A., Gungor, V.C. and Akan, O.B. (2013) A Cross-Layer QoS-Aware Communication Framework in Cognitive Radio Sensor Networks for Smart Grid Applications. IEEE Transactions on Industrial Informatics, 9, 1477-1485. http://dx.doi.org/10.1109/TII.2013.2242083

[14] Alwan, H. and Agarwal, A. (2013) Multi-Objective QoS Routing for Wireless Sensor Networks. International Conference on Computing, Networking and Communications (ICNC), San Diego, 1074-1079. http://dx.doi.org/10.1109/iccnc.2013.6504241

[15] Fang, X., Yang, D., Gundecha, P. and Xue, G. (2010) Multi-Constrained Anypath Routing in Wireless Mesh Networks. 7th Annual IEEE Communications Society Conference on Sensor, Mesh and Ad Hoc Communications and Networks (SECON), Boston, 1-9. http://dx.doi.org/10.1109/secon.2010.5508266

[16] Castalia Wireless Sensor Network Simulator. https://castalia.forge.nicta.com.au 


\section{Submit or recommend next manuscript to SCIRP and we will provide best service for you:}

Accepting pre-submission inquiries through Email, Facebook, LinkedIn, Twitter, etc.

A wide selection of journals (inclusive of 9 subjects, more than 200 journals)

Providing 24-hour high-quality service

User-friendly online submission system

Fair and swift peer-review system

Efficient typesetting and proofreading procedure

Display of the result of downloads and visits, as well as the number of cited articles

Maximum dissemination of your research work

Submit your manuscript at: http://papersubmission.scirp.org/ 